\title{
Distribution of zeros of the Husimi function in a realistic Hamiltonian molecular system
}

\author{
F. J. Arranz and F. Borondo \\ Departamento de Química, C-IX, Universidad Autónoma de Madrid, Cantoblanco 28049 Madrid, Spain \\ R. M. Benito \\ Departamento de Física y Mecánica, Escuela Técnica Superior de Ingenieros Agrónomos, Universidad Politécnica de Madrid, \\ 28040 Madrid, Spain \\ (Received 21 February 1996)
}

\begin{abstract}
In this paper we numerically check the validity of a theory on the distribution of zeros of the Husimi function due to Leboeuf and Voros [J. Phys. A 23, 1765 (1990)] for the integrability or chaoticity of a dynamical system in conditions which are not covered in the original proof. Our results for a generic Hamiltonian model for the LiCN molecule indicate also that in this case the conclusions of these authors hold. We have also found that this criterion is related to that proposed by Stratt, Handy and Miller based on the nodal complexity of the wave function [J. Chem. Phys. 71, 9911 (1979)]. Also, for scarred states there is a relation between the positions of the zeros and the fixed points of the Poincare map corresponding to the scarring periodic orbit, which is the main result of the paper. [S1063-651X(96)06409-4]
\end{abstract}

PACS number(s): 05.45+b, 03.65.Sq, 34.30.+h

\section{INTRODUCTION}

The correspondence between classical and quantum mechanics is a topic of renewed interest [1-4], specially in relation with the question of which is the quantum equivalent of classical chaos [5].

Chaos is a phenomenon well characterized from the classical point of view. Parameters such as the Lyapunov exponents [6], graphical representations such as the Poincaré surfaces of section [7], or the frequency analysis of the motion [8], are among the numerous tools that provide a detailed picture of the different regimes of a dynamical system. Nonlinear dynamics have firmly established that the best way to study chaos is to consider it within the framework of phase space [7]. With this approach this field has bloomed in the past two decades, giving rise to numerous important advances. On the other hand, the topic of "quantum chaos" is not so well developed and there are still many open questions in this field. Several criteria have been proposed for the existence of "quantum chaos," such as level spacings statistics [9], sensitivity of eigenvalues to changes in the Hamiltonian [10], or avoided crossings of energy levels [11]. Another one, which is very much related to the present work, is that of the nodal pattern complexity of wave functions [12]. According to it, states with a clear nodal pattern, in which the number of quanta in all degrees of freedom is clearly discernible, are classified as regular, while a complex nodal pattern is indicative of irregular or chaotic states. In this respect, the work of De Leon, Davis, and Heller [13] warned about the importance of considering classical resonances when doing this type of classification.

Other authors have considered the question of the quantum equivalent of classical chaos in the context of phase space. Much of this work has its start in an early paper of Wigner [14] who defined the following transform of the wave function

$$
W(\mathbf{P}, \mathbf{q})=(2 \pi \hbar)^{-D} \int \Psi^{*}(\mathbf{q}-\mathbf{x} / 2) \Psi(\mathbf{q}+\mathbf{x} / 2) e^{i \mathbf{P} \cdot \mathbf{x} / \hbar} d^{D} x
$$

as a quasiprobability density in phase space, being $D$ the number of degrees of freedom. Very early it was realized that the Wigner function suffers from the defect of not being positive definite [15]. In subsequent papers other authors solved this problem by defining, for example, functions suitably smoothed over regions of phase space of volume of the order of $\hbar^{D}$. Probably the most popular one is the Husimi function [16], which corresponds to a Gaussian smoothing and can be expressed in a very simple way

$$
H(\mathbf{P}, \mathbf{q})=(2 \pi \hbar)^{-D}|\langle z \mid \Psi\rangle|^{2}
$$

where $|z\rangle$ is a harmonic oscillator coherent state [17]. Due to Eq. (2), the Husimi function is also called coherent state representation, and it can be interpreted in a variety of ways [18]. Based in these two or other quasiprobability densities one can define quantum analoges (QSOS) to the classical Poincaré surface of section [19].

Many studies have been reported in the literature [20,21] based on the Husimi function for different systems, ranging from very simple analytical models to accurate molecular systems. The vast majority of them have concentrated on the maxima of the Husimi function, and discussed their relation with classical structures, mainly unstable periodic orbits. To refer to the influence of periodic orbits on the structure of the wave functions, the term "scarring" has been coined [22], and this is a topic that has received much attention in the literature. Scarring consists, as it is suggested by its name, in an accumulation of probability on periodic orbits, that constitute then a sort of backbone along which a sequence of perpendicular nodal planes (defining the corresponding quantum number) can be found. Obviously this has an effect in the QSOS, which in those cases shows maxima centered approximately on the corresponding fixed points.

Recently, Leboeuf, Voros, Cibils and others published a series of papers [23-25] in which they considered also the distribution of zeros of the Husimi function in different systems. In the first paper, Leboeuf and Voros [23] demon- 
strated that in the case of a quantum system having a twodimensional compact phase space, an arbitrary state of Hilbert space is completely defined by the zeros of the Husimi function. Moreover, for the eigenstates of the system, the distribution of zeros reflects, in the semiclassical limit, the underlying classical dynamics. If the system is classically integrable the zeros are localized on a line, while if the system behaves chaotically the zeros are spread all over the available phase space. In a subsequent paper [24], Leboeuf studied the time evolution of these zeros, considered as particles. He found that in chaotic systems the nonlinear terms induce interactions between the particles, leading them to uniformly cover all the available phase space, as it happens with its classical counterpart. In 1992 Cibils et al. [25] extended these studies to more general systems, and calculated the distribution of zeros for the spin-boson model [26]. This system presents significant differences with the class of systems considered by Leboeuf and Voros: in the first place the corresponding phase space for the spin-boson system is unbounded, and secondly, the dimension of that space is four instead of two. Considering QSOS, to overcome the second difference, these authors numerically checked that Leboeuf and Voros conclusions are also valid for this model. In all these three works it was emphasized the importance of considering the semiclassical limit, and accordingly a small value of the parameter $\hbar$ was used.

In this paper we investigate, in the same spirit of Cibils et al. the conjecture that Leboeuf and Voros result seems to be more general than suggested by the original proof. Accordingly, we have examined the distribution of zeros of the Husimi function for a realistic Hamiltonian model of the $\mathrm{LiCN}$ molecule. The organization of the paper is as follows. In the next section we describe the characteristics of the LiNC-LiCN molecular system used in our study and the calculations carried out. In Sec. III the wave function, QSOS and distribution of zeros for some representative states are presented and discussed, and finally our conclusions are summarized in the last section.

\section{SYSTEM AND CALCULATIONS}

The system that we have chosen to study corresponds to a realistic model for the LiNC-LiCN isomerizing system [27]. The vibrations of this molecule can be adequately studied by a two degrees of freedom model $(D=2)$, where the $\mathrm{CN}$ distance is kept frozen at its equilibrium value of $r_{e}=2.186$ a.u. The classical vibrational Hamiltonian $(J=0)$ is given in Jacobi coordinates by

$$
\mathcal{H}=\frac{P_{R}^{2}}{2 \mu_{1}}+\frac{1}{2}\left(\frac{1}{\mu_{1} R^{2}}+\frac{1}{\mu_{2} r_{e}^{2}}\right) P_{\theta}^{2}+V(R, \theta),
$$

where $R$ is the modulus of the vector from the $\mathrm{CN}$ center of mass to the $\mathrm{Li}$ nucleus, $r_{e}$ the modulus of the vector from nucleus $\mathrm{N}$ to nucleus $\mathrm{C}, \theta$ the angle formed by these two vectors, and $\mu_{1}$ and $\mu_{2}$ the corresponding $\mathrm{Li}-\mathrm{CN}$ and $\mathrm{C}-\mathrm{N}$ reduced masses.

The potential energy surface, $V(R, \theta)$, has been taken from the literature [28], and is shown in Fig. 1 as a contour plot. It presents two stable isomers at the linear configurations. The most stable one is LiNC that appears at

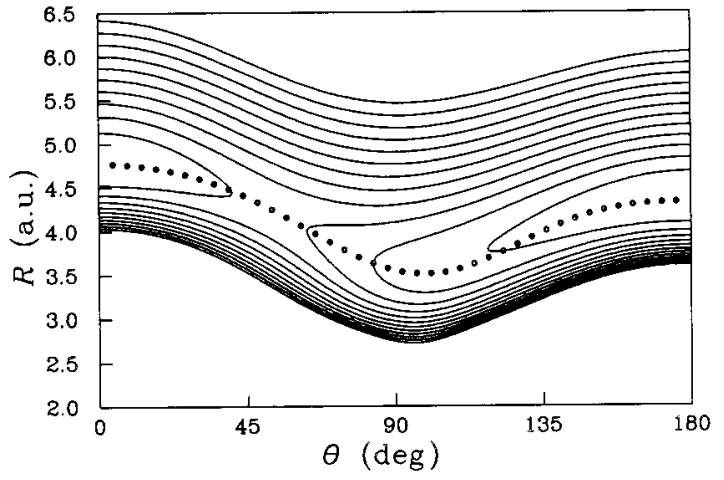

FIG. 1. Potential energy surface of the LiNC-LiCN isomerizing system. The minimum energy path connecting the two stable isomers, $\mathrm{LiCN}$ at $\theta=0^{\circ}$ and $\mathrm{LiNC}$ at $\theta=180^{\circ}$, is shown as a dotted line.

$\theta=180^{\circ}$, being the other $\mathrm{LiCN}$ located at $\theta=0^{\circ}$. The isomerization barrier is only $3454 \mathrm{~cm}^{-1}$; the $\mathrm{Li}$ atom can then easily perform large amplitude motions in the $\theta$ coordinate, sampling extensive regions of the potential energy surface. This causes that chaos sets in at low values of the excitation energy. Let us remark that although the calculation of Esser, Tennyson, and Wormer [28] is of relatively low level for actual standards in quantum chemistry [29], it is sufficiently realistic for the purpose of our dynamical research, since it contains many relevant molecular characteristics: anharmonicities, mode coupling, possibility of isomerization, etc.

Classical trajectories are calculated using a Gear algorithm for the numerical integration of the Hamilton equations of motion corresponding to Eq. (1). For each trajectory a Poincaré surface of section is computed by taking the minimum energy path $R_{e}(\theta)$ connecting the two isomers as the sectioning plane (see Fig. 1). This procedure gives the maximum dynamical information of the motion in the angular coordinate. Since $R_{e}$ depends on $\theta$, and in order to make the surface of section an area preserving map, it is necessary to make the following canonical transformation [21]

$$
\begin{gathered}
\rho=R-R_{e}(\theta), \quad \psi=\theta \\
P_{\rho}=P_{R}, \quad P_{\psi}=P_{\theta}+P_{R}\left[d R_{e}(\theta) / d \theta\right] .
\end{gathered}
$$

For the quantum calculations we have used the discrete variable representation-distributed Gaussian basis (DVRDGB) program of Bačić and Light [30] with a final basis set of 416 elements to obtain the first 100 vibrational states with the corresponding eigenvalues converged to within 0.01 $\mathrm{cm}^{-1}$. In our case, and in order to be able to compare with the classical results, we have also calculated Husimi based QSOS, $H_{Q S O S}$, using the same definition described above, i.e., $\rho=0$ and $P_{\rho}$ in a predetermined branch of the momentum function given by the energy conservation condition,

$$
H_{Q S O S}\left(\psi, P_{\psi}\right)=H\left[\psi, \rho=0, P_{\psi}, P_{\rho}=P_{\rho}(E)\right] .
$$

Moreover, $H_{Q S O S}$ (in the same way as $H$ ) is the squared modulus of a complex function $\Phi$, so that it can be written as

$$
H_{Q S O S}\left(\psi, P_{\psi}\right)=(2 \pi \hbar)^{-2}\left[\operatorname{Re}^{2} \Phi+\operatorname{Im}^{2} \Phi\right] .
$$



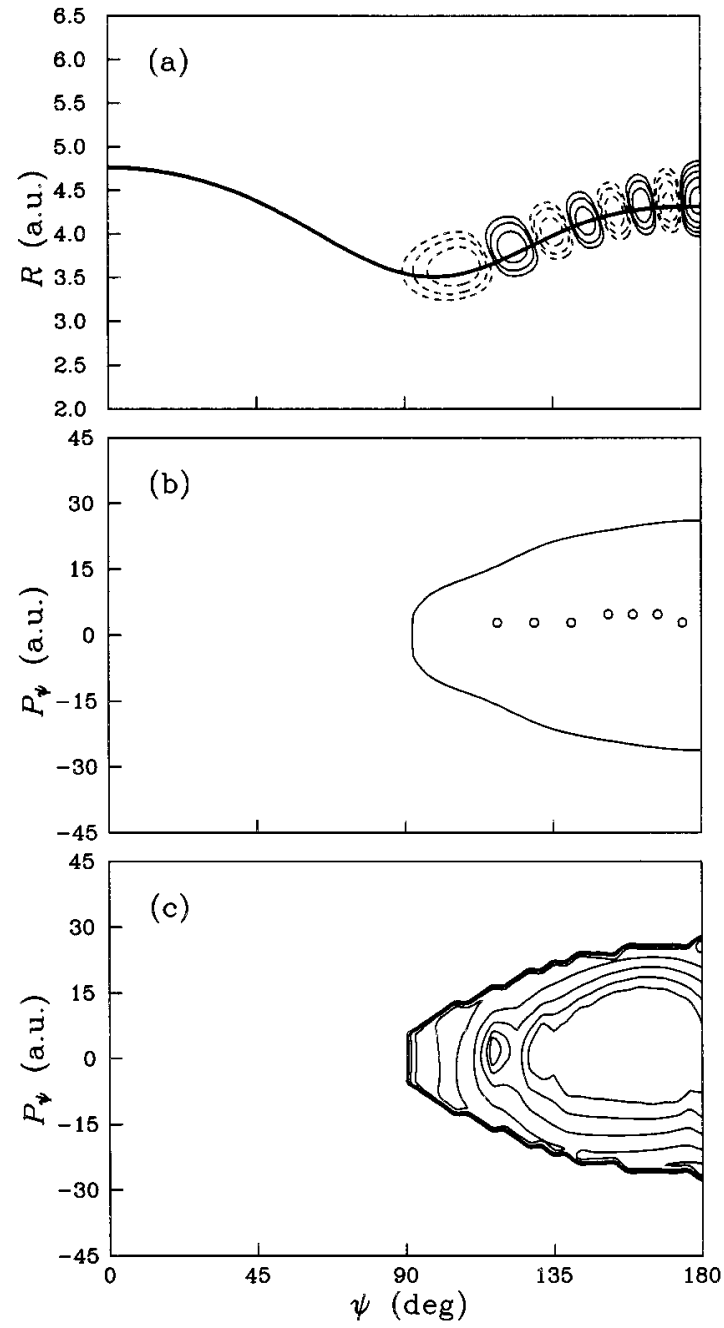

FIG. 2. (a) Wave function, (b) zeros, and (c) quantum surface of a section for the regular state $N=11$. Contours lines are drawn at $4 \%, 8 \%, 16 \%, 32 \%$, and $64 \%$ of the maximum amplitudes of the functions. Those which are solid represent positive amplitude while the dashed represent negative amplitude. The minimum energy path of the potential energy surface has also been included.

The QSOS defined in this way has been numerically calculated and full details will be given elsewhere [31]. Due to the fact that $\Phi$ is not an analytic complex function, the technique used by Cibils et al. to calculate the zeros of the Husimi function is not directly applicable. Accordingly we have used a suitable modification devised by us. The available $\left(\psi, P_{\psi}\right)$ phase space plane is divided into square cells, and then the number of zeros contained in each cell calculated. This is done in the vector field associated to the QSOS according to

$$
\left(\psi, P_{\psi}\right) \stackrel{\text { T }}{\rightarrow}(\operatorname{Re} \Phi, \operatorname{Im} \Phi),
$$

using the fact that the index of a curve [32] is equal to the number of zeros contained in the area enclosed by it [33]. In our case the index $\mathcal{I}$ is defined as

$$
\mathcal{I}(\gamma)=\frac{1}{2 \pi} \oint_{\gamma} d\left[\tan ^{-1}\left(\frac{\operatorname{Im} \Phi}{\operatorname{Re} \Phi}\right)\right] .
$$
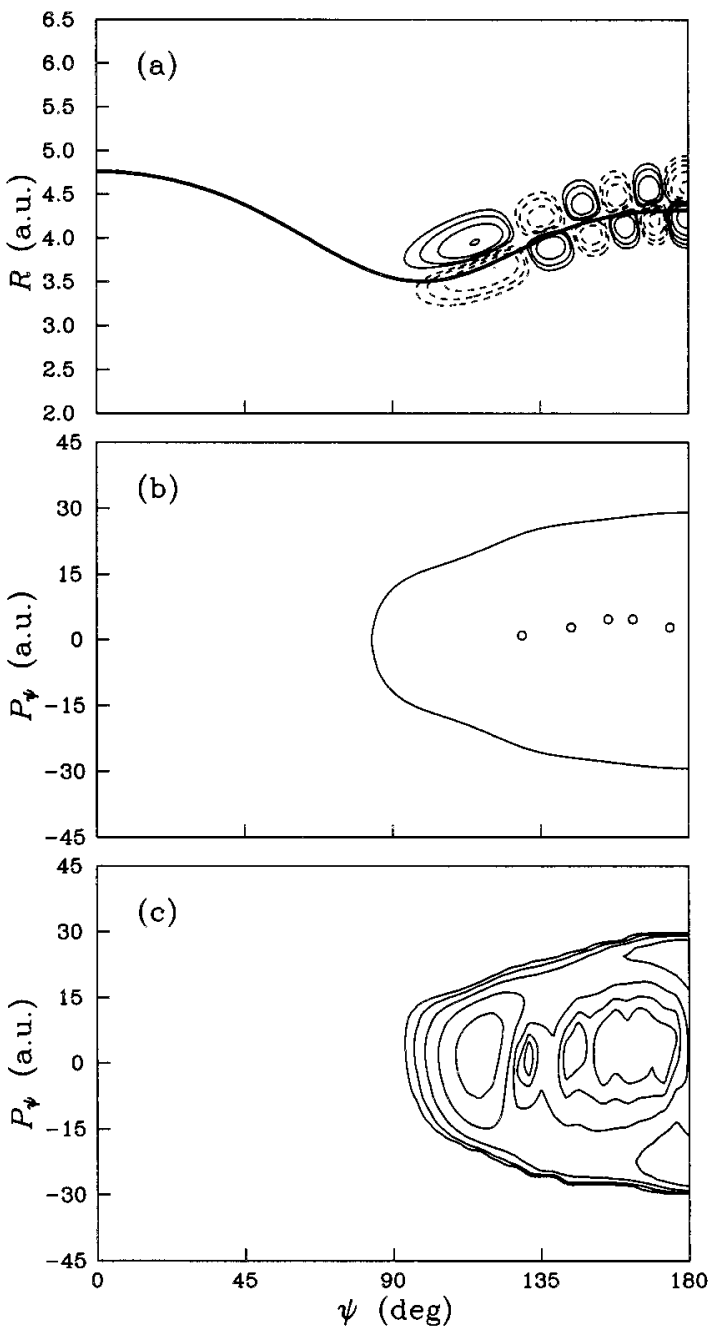

FIG. 3. Same as Fig. 2 for the regular state $N=17$.

Note that this is an extension to applications $R^{2} \rightarrow R^{2}$ of the well known principle of the argument of complex variable that holds in applications $\mathrm{C} \rightarrow \mathrm{C}$ [34].

To conclude this section we would like to point out that the computer effort involved in the calculation of the zeros of the QSOS is not small in our case, since the integration required for the calculation of the Husimi function is done numerically [31]. The calculation of each point in the $\left(\psi, P_{\psi}\right)$ plane takes 7.5 seconds in an IBM Risc 6000 workstation, and to characterize each zero not less than 736 points are required (the actual number of points depends on the indeterminacies encountered) [33].

\section{RESULTS AND DISCUSSION}

In this section we discuss the results concerning the first 100 quantum eigenstates of the LiNC-LiCN system. In Fig. 2 we show the wave function, zeros and QSOS of state $N=11$ (in what follows $N$ will be used as the state number starting from $N=1$ for the ground state). It is seen that this state presents a well defined wave function nodal structure, corresponding to the quantum numbers $\left(n_{\rho}, n_{\psi}\right)=(0,14)$. Since it is excited solely in the angular coordinate all maxima in the QSOS are located close to the border of the classically avail- 


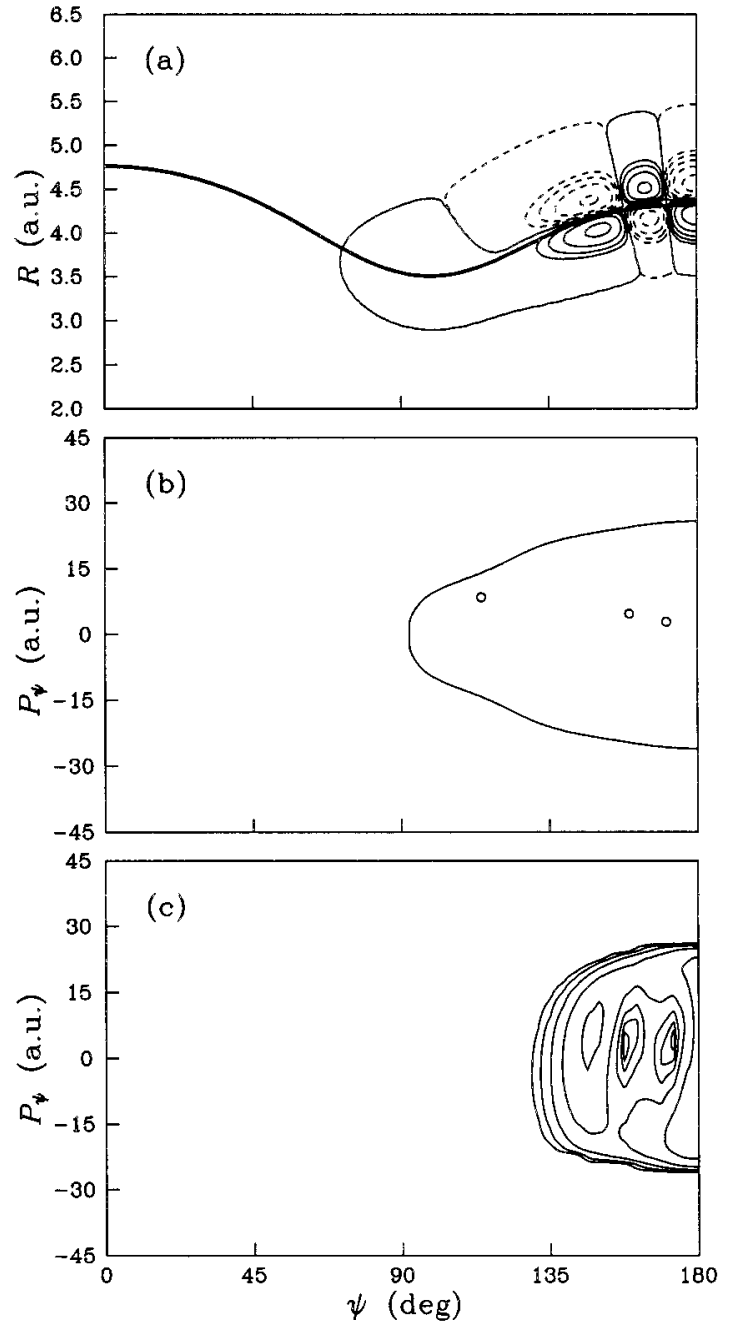

FIG. 4. Same as Fig. 2 for the state $N=10$. An extra contour line representing $10^{-4} \%$ of the maximum amplitude of the wave function has been included.

able phase space area as can be appreciated in the figure. Moreover, the zeros are aligned along a curve lying very close to the $P_{\psi}=0$ axis. This result is in perfect agreement with the conclusions of Refs. [23-25], which is not unexpected since state $N=11$ is clearly regular. Let us consider next the relationship existing between distribution of zeros and wave function nodal structure. By comparing of parts (a) and (b) of Fig. 2 it is apparent that there are as many zeros as nodal planes and that the location of them (approximately) coincides. This result is very interesting since it implies a connection between Leboeuf and Voros criterion which is based on phase space considerations, and Stratt, Handy, and Miller criterion which is based on configuration space considerations. Let us remark that the above result is characteristic of anharmonic systems, since in the case of a harmonic oscillator all zeros accumulate at the origin of coordinates.

A second example is presented in Fig. 3, where the results corresponding to the $N=17$ quantum state are shown. According to the nodal structure complexity criterion it corresponds to a regular state with quantum numbers $\left(n_{\rho}, n_{\psi}\right)=(1,10)$. In this case there is excitation in the $\rho$ coordinate, which reflects in the fact that the maxima of the QSOS are not located around the border of the available
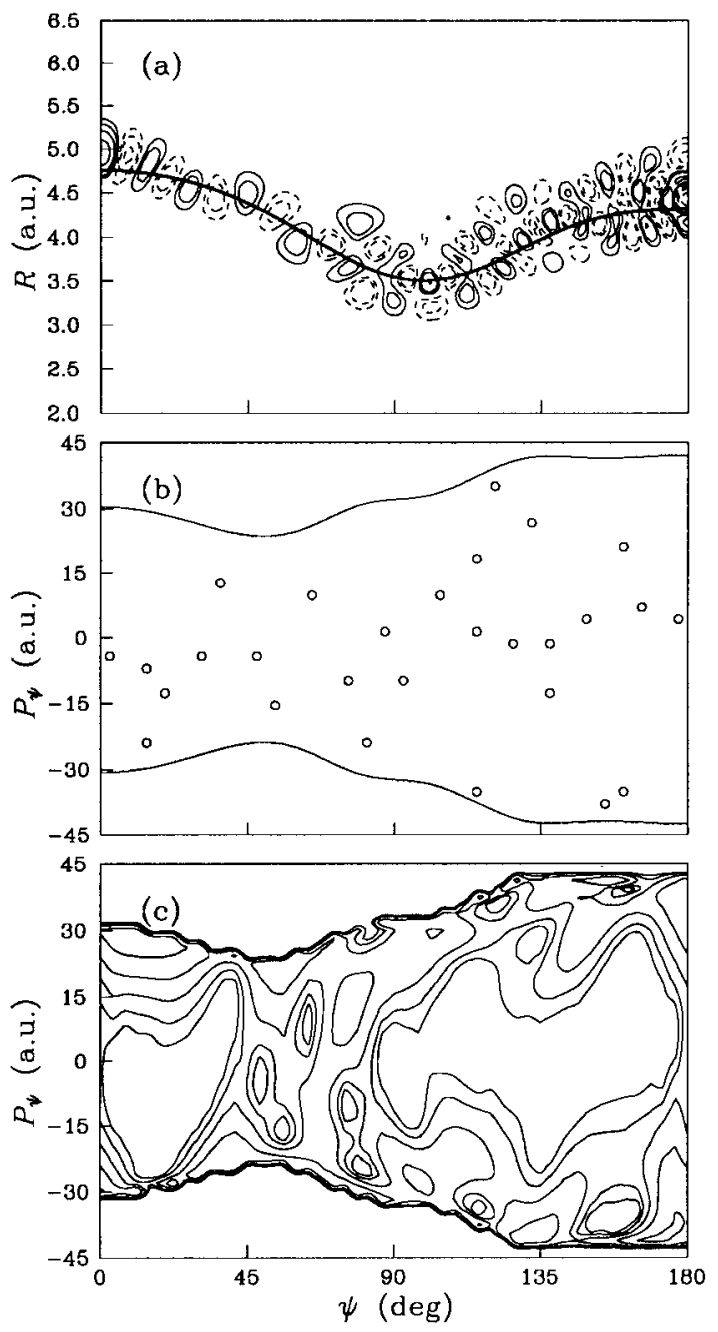

FIG. 5. Same as Fig. 2 for the irregular state $N=99$.

phase space, but more towards the inner part. However, this does not affect the structure of the distribution of zeros, which is similar to that found in the previous case. Obviously, the information about the excitation in $\rho$ is contained in the other Poincare surface of section, i.e., $H_{Q S O S}\left(\rho, P_{\rho}\right)$ corresponding to $\psi=180^{\circ}$ for the LiNC isomer or $\psi=0^{\circ}$ for the LiCN isomer. Similar results have been obtained for all states in our calculation which present a well defined nodal pattern.

The criterion of the wave function nodal structure complexity is necessarily qualitative. In particular, the number of nodes is very sensitive to the wave function amplitude level which is considered. In general, one focuses on the high values of the amplitude [12] where the pattern is more clear. But when low levels are considered a much more complicated nodal structure might emerge. A good example of this is state number ten, which is shown in Fig. 4. In the part corresponding to the wave function [panel (a)] we have included, in addition to the contour levels used in the previous figures a low contour corresponding to $10^{-4} \%$ of the maximum amplitude. According to the structure of these contours the classification is a little uncertain. If one just takes into account the high level contours, the wave function presents a well defined nodal pattern corresponding to state 

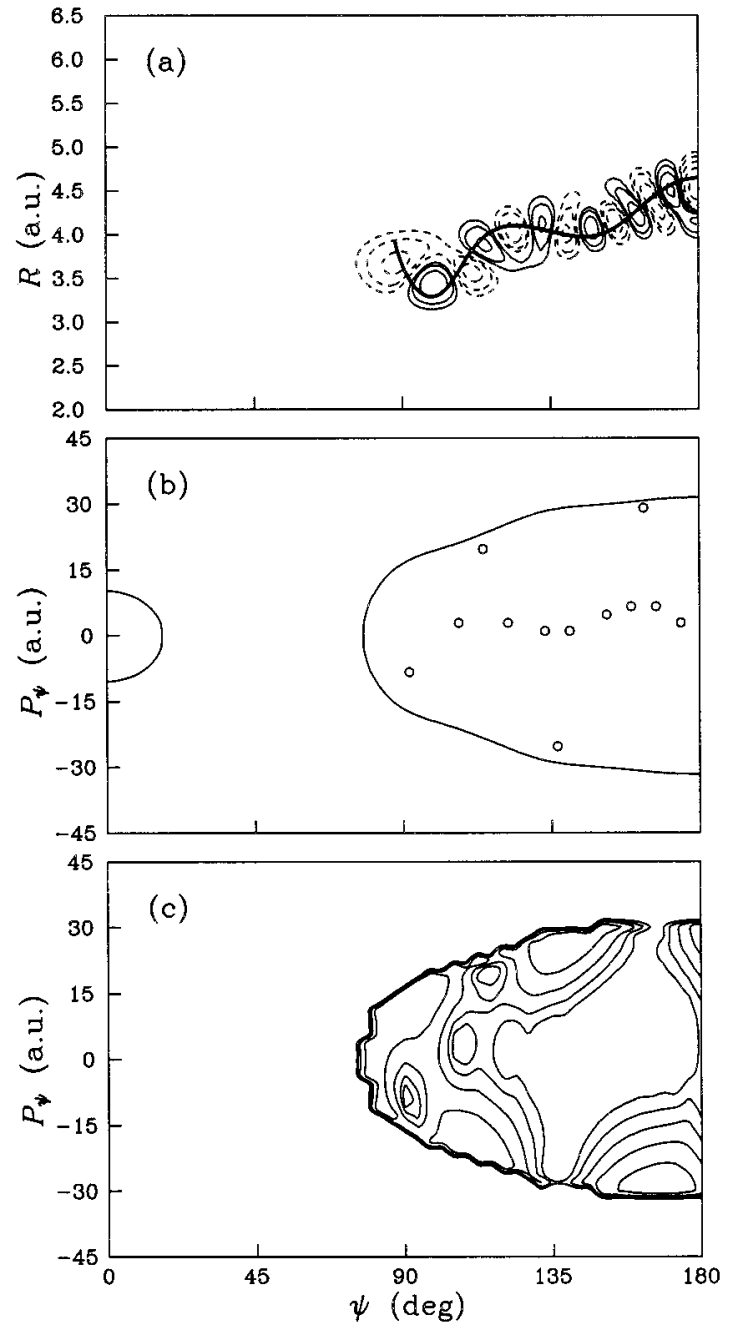

FIG. 6. Same as Fig. 2 for the scarred state $N=24$. In panel (a) we have plotted, superimposed, the scarring periodic orbit (instead of the minimum energy path).

$\left(n_{\rho}, n_{\psi}\right)=(1,4)$. However, when the low level contour is considered an extra, not very well defined, seminode appears at $\theta \simeq 117^{\circ}$. In panel (b) it is seen that at the position of the seminode there is a zero, that in this case is not close to the $P_{\psi}=0$ axis. This result indicates that the distribution of zeros constitutes a very sensitive criterion for analyzing the nodal pattern complexity.

A lot of caution must be exerted when considering low contours of the wave functions, since they ultimately reflect the structure of the basis set functions. In our case we have checked the robustness of our conclusions by repeating selected calculations with a basis set of 2016 elements which gives 900 converged eigenvalues.

In Fig. 5 the results corresponding to state $N=99$ are shown. The nodal pattern is clearly irregular, and the zeros are spread uniformly over the available phase space, again in agreement with Leboeuf and Voros result.

Let us point out that all calculations that we are reporting here have been obtained for $\hbar=1$ a.u.. Thus the results seem to indicate that this value of $\hbar$ is low enough in our system to allow for this type of studies. On the other hand changing the value of $\hbar$ in a generic Hamiltonian like ours would introduce additional complications. For example, lowering $\hbar$ in-

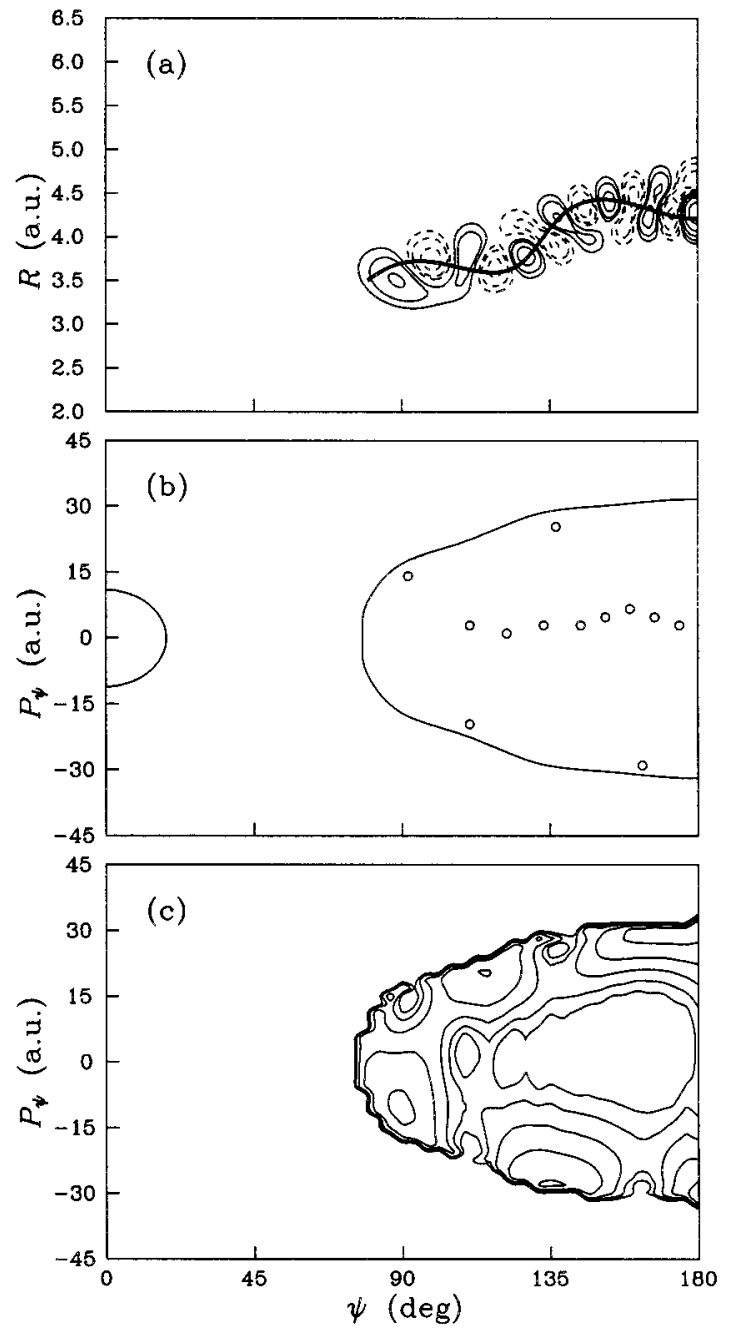

FIG. 7. Same as Fig. 6 for the scarred state $N=25$.

creases the number of states that can be "accommodated" in the regular region (see Fig. 8 below). Moreover, since LiNCLiCN has a potential surface with two separate wells, with states that behave differently with $\hbar$, changing this parameter causes the appearance of a complicated series of avoided crossings, some isolated and others overlapping [11]. The disentanglement of this problem is not easy, and this is a task that we are performing at present. Some preliminary results have been published in Ref. [35], in which we showed how a transition from chaos (scarred states) to regularity takes place as $\hbar$ is decreased.

As we stated in the Introduction the criterion of nodal pattern complexity presents some problems when there are classical resonances in the system [13]. In this case very often some wave functions appear scarred by any of the two periodic orbits, one stable and the other unstable, corresponding to the resonance. In the rest of the discussion we will refer to these two periodic orbits as complementary in the sense that they come from the same unperturbed resonant invariant torus [7].

The LiNC-LiCN system, due to its dynamical complexity, presents a great number of resonances even at modest excitation energies, and we have presented a systematic study of the corresponding bifurcation diagrams in Ref. [36]. Among 


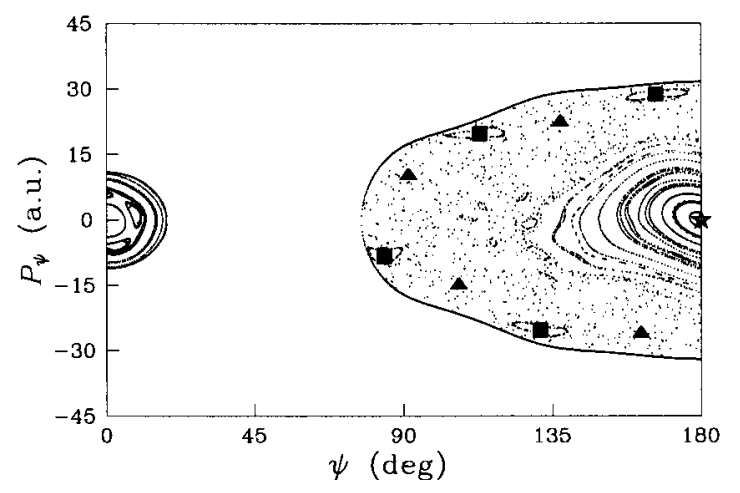

FIG. 8. Poincaré surface of a section for the LiNC-LiCN system at an energy of $2568.03 \mathrm{~cm}^{-1}$. We have marked the elliptic fixed point corresponding to the linear $\left(\psi=\theta=180^{\circ}\right)$ LiNC isomer with a star, and the elliptic and hyperbolic fixed points, correponding to the resonance discussed in the text, with squares and triangles.

of all them there is one resonance, belonging to the principal family, that has a profound influence in the quantum mechanics of this system in the range of energies considered in this paper (up to $4610 \mathrm{~cm}^{-1}$ ). The associated unstable and stable periodic orbits are shown in Figs. 6(a) and 7(a) respectively. In Fig. 8 we also present a composite Poincaré surface of section, calculated at an energy of $2568.03 \mathrm{~cm}^{-1}$ (intermediate between the eigenvalues of states 24 and 25), in which the corresponding fixed points are clearly visible (triangles and squares, respectively) in the middle of a chaotic sea. From Figs. 6(a) and 7(a), it is apparent that these orbits "scar" the wave functions of states $N=24$ and $N=25$. This is also appreciated in Figs. 6(c) and 7(c), where it is seen that the corresponding QSOS present maxima centered on the classical fixed points (see Fig. 8). The examination of the distribution of zeros in Figs. 6(b) and 7(b) reveals some interesting results. In the first place, and similarly to what is obtained for regular states, in both cases there are as many zeros as nodal planes (that in this case must be counted along the periodic orbit). Secondly, and more important, two types of zeros are obtained: some of them appear distributed along a curve close to the $P_{\psi}=0$ axis, again as for a regular state, while the rest localize over the fixed points of the periodic orbit complementary to the one "scarring" the state.

\section{CONCLUSIONS}

In this paper we have presented a quantum study in phase space of the vibrational states of a realistic model for the molecular isomerizing system LiNC-LiCN for excitation energies up to $4610 \mathrm{~cm}^{-1}$. Due to the characteristics of the potential energy surface, the $\mathrm{Li}$ atom can execute large amplitude motions in the bending coordinate. This causes the system to present a very chaotic behavior, even at low vibrational energies. This study has been carried out by numerically calculating QSOS based on the Husimi function. Although the maxima of these QSOS contain information about the correspondence between classical and quantum mechanics, in this paper we have focused on the corresponding distribution of zeros.

The distribution of zeros of the Husimi function as a criterion for chaos in quantum mechanics is relatively new [23] and consequently has not received much attention in the literature yet. Leboeuf and Voros found that for a particular class of systems these zeros appear localized on a line for classically integrable systems, while they spread all over the available phase space for systems that behave chaotically.

In this paper we have investigated the validity of the conclusion of Leboeuf and Voros conclusions in generic Hamiltonian systems. Our numerical calculations for the LiNCLiCN molecule indicate that this seems to be the case. Moreover, we have also seen that this criterion is related to that of Stratt, Handy, and Miller [12] based on the nodal complexity of the wave functions. Specifically, we have seen that the number of zeros is equal to the number of nodes of the wave function (even if they are not completely well defined) and that their positions coincide. Finally, and we believe that this constitutes the main contribution of this paper, for states which are scarred by periodic orbits some zeros appear localized on the fixed points of the Poincare map of the complementary (in the sense of stable-unstable) periodic orbit.

\section{ACKNOWLEDGMENTS}

This work has been supported in part by DGICYT (Spain) under Contract PB92-0181. F.J.A. gratefully acknowledges a grant from the UAM.
[1] A. M. Ozorio de Almeida, Hamiltonian Systems: Chaos and Quantization (Cambridge University Press, Cambridge, 1988).

[2] M. C. Gutzwiller, Chaos in Classical and Quantum Mechanics (Springer, New York, 1990).

[3] L. E. Reichl, The Transition to Chaos in Conservative Classical Systems: Quantum Manifestations (Springer-Verlag, New York, 1992).

[4] K. Nakamura, Quantum Chaos. A New Paradigm of Nonlinear Dynamics (Cambridge University Press, Cambridge, 1993).

[5] M. V. Berry, Proc. R. Soc. London Ser. A 423, 219 (1989).

[6] A. H. Nayfeh and B. Balachandran, Applied Nonlinear Dynamics (Wiley, New York, 1995), pp. 525-537.

[7] A. J. Lichtenberg and M. A. Lieberman, Regular and Stochas- tic Motion (Springer, Berlin, 1983).

[8] J. Laskar, Physica D 67, 257 (1993).

[9] O. Bohigas and M. J. Giannoni, in Mathematical and Computational Methods in Nuclear Physics, edited by J. S. Dehesa, J. M. G. Gomez and A. Polls (Springer-Verlag, New York, 1984); T. A. Brody, J. Flores, J. B. French, P. A. Mello, A. Pandey, and S. S. M. Wong, Rev. Mod. Phys. 53, 385 (1981).

[10] N. Pomphrey, J. Phys. B 7, 1909 (1974); F. von Oppen, Phys. Rev. Lett. 73, 798 (1994).

[11] D. W. Noid, M. L. Koszykowski, and R. A. Marcus, Chem. Phys. Lett. 73, 269 (1980); D. W. Noid, M. L. Koszykowski, M. Tabor, and R. A. Marcus, J. Chem. Phys. 72, 6169 (1980); T. Takami, Phys. Rev. Lett. 68, 3371 (1992); Phys. Rev. E 52, 2434 (1995). 
[12] R. M. Stratt, N. C. Handy, and W. H. Miller, J. Chem. Phys. 71, 9911 (1979).

[13] N. De Leon, M. J. Davis, and E. J. Heller, J. Chem. Phys. 80, 794 (1984).

[14] E. P. Wigner, Phys. Rev. 40, 749 (1932).

[15] N. D. Cartwright, Physica A 83, 210 (1976).

[16] K. Husimi, Proc. Phys. Math. Soc. Jpn. 22, 264 (1940).

[17] A. Perelomov, Generalized Coherent States and Their Applications (Springer, New York, 1986).

[18] J. E. Harriman, J. Chem. Phys. 88, 6399 (1988); K. Takahashi, Prog. Theor. Phys. Suppl. 98, 109 (1989).

[19] P. Leboeuf and M. Saraceno, J. Phys. A 231745 (1990); Phys. Rev. A 41, 4614 (1990).

[20] K. Takahashi and N. Saito, Phys. Rev. Lett. 55, 645 (1985); K. Takahashi, J. Phys. Soc. Jpn. 55, 762 (1986); 55, 1443 (1986); 55, 1783 (1986); K. Zyczkowski, Phys. Rev. A 35, 3546 (1987); M. J. Davis, J. Phys. Chem. 92, 3124 (1988); J. L. Anchell and J. E. Harriman, J. Chem. Phys. 89, 6860 (1988); G. Radons and R. E. Prange, Phys. Rev. Lett. 61, 1691 (1988); R. L. Waterland, J. Yuan, C. C. Martens, R. E. Gillilan, and W. P. Reinhardt, Phys. Rev. Lett. 61, 2799 (1988); C. C. Martens, J. Chem. Phys. 90, 7065 (1989); J. L. Anchell, J. Chem. Phys. 92, 4342 (1990); R. C. Morrison and R. G. Parr, Int. J. Quantum Chem. 39, 823 (1991); D. Lalovic, D. M. Davidovic, and N. Bijedic, Phys. Lett. A 166, 99 (1992); R. Scharf and B. Sundaran, Phys. Rev. A 45, 3615 (1992); W. A. Lin and L. E. Ballentine, Phys. Rev. A 45, 3637 (1992); A. P. Jalil and E. Yurtsever, Tr. J. Phys. 18, 1095 (1994); E. Yurtsever, Phys. Rev. E 50, 3422 (1994); W. Schweizer, M. Schaich, W. Jans, and H. Ruder, Phys. Lett. A 189, 64 (1994), G. G. de Polavieja, F. Borondo, and R. M. Benito, Int. J. Quantum Chem. 51, 555 (1994).

[21] R. M. Benito, F. Borondo, J. H. Kim, B. G. Sumpter and G. S. Ezra, Chem. Phys. Lett. 161, 60 (1989).

[22] E. J. Heller, in Chaos and Quantum Physics, edited by M. Giannoni, A. Voros, and J. Zinn-Justin (Elsevier, Amsterdam, 1991).
[23] P. Leboeuf and A. Voros, J. Phys A 23, 1765 (1990).

[24] P. Leboeuf, J. Phys A 24, 4575 (1991).

[25] M. B. Cibils, Y. Cuche, P. Leboeuf, and W. F. Wreszinki, Phys. Rev. A 46, 4560 (1992).

[26] See, for example, R. Graham and M. Hohnerbach, in Quantum Measurement and Chaos, edited by E. R. Pike and S. Sarkar (Plenum, New York, 1987), NATO-ASI series B, vol. 161, pp. 147-162.

[27] F. Borondo and R. M. Benito, in Frontiers of Chemical Dynamics, Vol. 470 of NATO-Advanced Study Institite Series C; Mathematical and Physical Sciences, edited by E. Yurtsever (Kluwer, Dordrecht, 1995), pp. 371-392.

[28] R. Esser, J. Tennyson, and P.E.S. Wormer, Chem. Phys. Lett. 89, 223 (1982).

[29] J. Makarewicz and T.-K. Ha, Chem. Phys. Lett. 232, 497 (1995).

[30] Z. Bačić and J. Light, Ann. Rev. Phys. Chem. 40, 469 (1989).

[31] R. M. Benito and F. Borondo (unpublished).

[32] V. I. Arnold, Ordinary Differential Equations (Springer, Berlin, 1992).

[33] This is not strictly true since the integrand in Eq. (8) can rotate in both senses leading to cancellations in the index. (Note, that again, this is due to the fact that $\Phi$ is not an analytic complex function). Also some numerical indeterminacies arise when the zeros are located very close to the border. These two problems have been prevented in our calculations by checking the consistency of our results against changes in the calculation procedure.

[34] R. V. Churchill and J. W. Brown, Complex Variable and Applications (McGraw-Hill, New York, 1990).

[35] F. J. Arranz, F. Borondo, and R. M. Benito, J. Chem. Phys. 104, 6401 (1996).

[36] F. Borondo, A. A. Zembekov, and R. M. Benito, Chem. Phys. Lett. 246, 421 (1995); R. Prosmiti, S. C. Farantos, R. Guantes, F. Borondo, and R. M. Benito, J. Chem. Phys. 104, 2921 (1996). 